\title{
SUPER-RIGID FAMILIES OF STRONGLY BLACKWELL SPACES
}

\author{
MANFRED DROSTE
}

(Communicated by R. Daniel Mauldin)

\begin{abstract}
We construct a complete subfield $\mathscr{F}$ of $\mathscr{P}(\mathbf{R})$, isomorphic to $\mathscr{P}(\mathbf{R})$, of pairwise non-Borel-isomorphic rigid strong Blackwell subsets of $\mathbf{R}$ such that there are only 'very few' measurable functions between any two members of $\mathscr{F}$. As a consequence, we obtain large chains and antichains of non-isomorphic rigid strong Blackwell subsets of $\mathbf{R}$. Also, there is a collection of continuously many dense subsets of $\mathbf{R}$ such that any two of them differ only by two elements, but none of them is a continuous image of any other.
\end{abstract}

1. Introduction. In this paper, we will construct a large subfield $\mathscr{F}$ of $\mathscr{P}(\mathbf{R})$ with the following properties. Any two members $A, B \in \mathscr{F}$ which are nonempty proper subsets of $\mathbf{R}$ will be nonanalytic strong Blackwell sets (definitions below) with only 'very few' measurable functions both from $A$ into $B$ (or vice versa) or from $A$ into itself. In particular, the spaces $\left(A, \mathscr{B}_{A}\right)$ and $\left(B, \mathscr{B}_{B}\right)$ (both sets equipped with the natural Borel structure) will be nonisomorphic if $A \neq B$, and $\left(A, \mathscr{B}_{A}\right)$ will be a rigid Borel space in the sense of [1], i.e., any isomorphism of $\left(A, \mathscr{B}_{A}\right)$ onto itself moves at most countably many points. In fact, we show that for any topologically sufficiently large Blackwell subset $X$ of $\mathbf{R}$, there exists a complete subfield $\mathscr{F}$ of $\mathscr{P}(X)$ with the above properties.

Let $\mathscr{A}$ be a separable $\sigma$-algebra on a set $A$, i.e. $\mathscr{A}$ is countably generated and contains all singletons. Then $(A, \mathscr{A})$ is a Blackwell space, if the only separable substructure of $\mathscr{A}$ is $\mathscr{A}$ itself. Also, $(A, \mathscr{A})$ is a strong Blackwell space if any two countably generated sub- $\sigma$-algebras of $\mathscr{A}$ with the same atoms coincide. Subsets of $\mathbf{R}$ will always be endowed with the natural Borel- $\sigma$-field, and we say that a subset $A$ of $\mathbf{R}$ is a (strong) Blackwell set if $\left(A, \mathscr{B}_{A}\right)$ is a (strong) Blackwell space. Ramachandran [11] discusses strong Blackwell spaces as a natural model for probability theory on which various concepts of independence of random variables coincide. Already Blackwell [3] and also Mackey [9], in a study of group representations, showed that any analytic subset of the reals is a (strong) Blackwell set. Answering a question of Blackwell [3], Orkin [10] constructed a nonanalytic Blackwell subset of $\mathbf{R}$. Ryll-Nardzewski [13] (see also [1]) found a subset $X$ of $\mathbf{R}$ such that both $X$ and $Y=\mathbf{R} \backslash X$ are nonanalytic strong Blackwell sets; by a recent result of Shortt [16], $\left(X, \mathscr{B}_{X}\right)$ and $\left(Y, \mathscr{B}_{Y}\right)$ must then be nonisomorphic. On the other hand, Bhaskara Rao and Rao [1] constructed a subset $X$ of $\mathbf{R}$ such that $\left(X, \mathscr{B}_{X}\right)$ is a rigid Borel space. In the present paper, we wish to sharpen these results as indicated qualitatively above. Shortt [14] calls a subset $X$ of $\mathbf{R}$ Borel-dense, if $X$ intersects each

Received by the editors May 26, 1987.

1980 Mathematics Subject Classification (1985 Revision). Primary 28A20; Secondary 28A05, 60A99, 54C05.

Key words and phrases. Blackwell space, strong Blackwell space, rigid Borel space, separable space, measurable function. 
uncountable Borel set of $\mathbf{R}$, and shows that under the assumption of Borel-density various Blackwell concepts coincide; in particular any Borel-dense Blackwell subset $X$ of $\mathbf{R}$ is strongly Blackwell. In fact, many of the strongly Blackwell spaces constructed in the literature can be shown to be Borel-dense in some suitable Polish space and hence without loss of generality in $R$, cf. $[1,2,5-7,10,13-15]$.

Before stating our main result, let us introduce some more notation. Let $X \subseteq \mathbf{R}$ and $f: X \rightarrow \mathbf{R}$ be a measurable function. Then $\operatorname{supp}(f)=\{x \in X: x \neq f(x)\}$, the support of $f$. We call $f$ inessential, if $f(\operatorname{supp}(f))$ is countable, and otherwise $f$ is essential. (For the origin of this notion (in a different context) we refer the reader to Dugas and Göbel [4, p. 458].) As there are always many inessential measurable functions acting on $X$, we can only hope to construct subsets $X$ of $\mathbf{R}$ with no essential measurable mappings. Observe in the following that if $f$ has uncountable support and, for instance, either $f$ is injective or $f$ maps $X$ onto $X$, then $f$ is essential. A family $\mathscr{F}$ of subsets of $\mathbf{R}$ will be called super-rigid if for any two members $A, B \in \mathscr{F}$ there is no essential measurable mapping $f$ of $A$ into $B$. Also, we say that a subset $A$ of $\mathbf{R}$ is super-rigid if $\{A\}$ is super-rigid; then in particular $\left(A, \mathscr{B}_{A}\right)$ is rigid. A subfield $\mathscr{F}$ of $\mathscr{P}(X)$ is complete, if it is closed under arbitrary unions. We will show:

THEOREM 1. Let $X$ be any Borel-dense Blackwell subset of $\mathbf{R}$. There exists a complete subfield $\mathscr{F}$ of $\mathscr{P}(X)$ with the following properties:

(1) Each non-empty set $A \in \mathscr{F}$ is a Borel-dense strong Blackwell set.

(2) $\mathscr{F} \backslash\{\varnothing, X\}$ is super-rigid.

(3) $\mathscr{F}$ is isomorphic (as a Boolean algebra) to $\mathscr{P}(\mathbf{R})$.

Note that in particular $X=\mathbf{R}$ satisfies the hypothesis of Theorem 1 . We list a few immediate consequences of Theorem 1 . Let $A, B \in \mathscr{F}$ be nonempty proper subsets of $X$.

(1) Both $A$ and $X \backslash A$ are nonanalytic (in fact, universally nonmeasurable). If $A \nsubseteq B$, there is no measurable injection $f: A \rightarrow B$ and also no measurable mapping of $B$ onto $A$, as $A \backslash B \in \mathscr{F}$ is uncountable. Whenever $f: A \rightarrow B$ is an order-preserving injection, then $A \subseteq B$ and $f$ is the identity. Hence Theorem 1 contains a classical result of Sierpiński [17] (cf. also [12, §9.2]) on rigid dense order types contained in $(\mathbf{R}, \leq)$. Moreover, if $f: A \rightarrow A$ is onto and nondecreasing, then $f$ is again the identity, showing that $(A, \leq)$ is a Hopfian order in the sense of Ash $[12$, p. 155].

(2) Let $P, Q$ be any two nonatomic measures on $\left(A, \mathscr{B}_{A}\right)$ such that $Q=f(P)$ for some measurable mapping $f$ of $A$ into itself. As $f$ is inessential, it follows that $P=Q$.

(3) If $A$ and $B$ are disjoint, there are no two nonatomic measures $P$ and $Q$ on $\left(A, \mathscr{B}_{A}\right)$ and $\left(B, \mathscr{B}_{B}\right)$, respectively, such that $Q$ is the image of $P$ under some measurable mapping of $A$ into $B$.

(4) Let $c$ denote the cardinality of the continuum. $\left\{\mathscr{B}_{A}: A \in \mathscr{F} \backslash\{\varnothing, X\}\right\}$ constitutes a family of $2^{c}$ pairwise nonisomorphic countably generated $\sigma$-algebras. Clearly, $2^{c}$ is the maximal possible size of such a family.

Further consequences of Theorem 1 are given in $\S 2$.

2. Proof of Theorem 1 and further consequences. In this section we wish to prove Theorem 1 and derive a few further consequences from it. The following 
notions developed by R. M. Shortt (see, e.g., $[2,14])$ will be useful for us. A subset $X$ of $\mathbf{R}$ is called Borel-dense (of order 1 ) in $\mathbf{R}$ if $X$ intersects each uncountable member of $\mathscr{B}_{\mathbf{R}}$. Any such set $X$ has cardinality $c$. A subset $R$ of $\mathbf{R} \times \mathbf{R}$ is reticulate if $R \subseteq(C \times \mathbf{R}) \cup(\mathbf{R} \times C)$ for some countable subset $C \subseteq \mathbf{R}$. A subset $X$ or $\mathbf{R}$ is Borel-dense of order 2 if $X \times X$ intersects every set $R$ in $\mathscr{B}_{\mathbf{R} \times \mathbf{R}}$ which is not reticulate; in this case $X$ is Borel-dense of order 1 . We also have, among others, the following equivalences:

LEMMA 2.1 (SHORTT [14]). For any subset $X$ of $\mathbf{R}$ the following are equivalent:

(1) $X$ is Borel-dense of order 2 in $\mathbf{R}$.

(2) $X$ is Borel-dense and a Blackwell set.

(3) $X$ is Borel-dense and a strong Blackwell set.

As usual we identify each cardinal with the least ordinal of its cardinality. We first need a few preparations.

LEMMA 2.2. Let $X$ be a Borel-dense subset of $\mathbf{R}$ and $f: \mathbf{R} \rightarrow \mathbf{R}$ an essential measurable function. There exists a subset $A$ of $X \cap \operatorname{supp}(f)$ of cardinality $c$ such that $f$ acts injectively on $A$.

Proof. Let $S=\operatorname{supp}(f)=\{x \in \mathbf{R}: x \neq f(x)\}, f^{\prime}$ be the restriction of $f$ to $S$, and $T=f(S)=f^{\prime}(S)$. Thus $T$ is uncountable and analytic. Let $T^{*}$ be the set of all $t \in T$ for which $f^{\prime-1}(\{t\})$ is uncountable. Then $T^{*}$ is analytic (see [8, p. 496]). If $T^{*}$ is uncountable, choose for each $t \in T^{*}$ an element $x_{t} \in X \cap f^{\prime-1}(\{t\})$ and put $A=\left\{x_{t}: t \in T^{*}\right\}$. Then $|A|=\left|T^{*}\right|=c$. On the other hand, if $T^{*}$ is countable, $T \backslash T^{*}$ is uncountable and analytic and hence $\left|X \cap f^{\prime-1}\left(T \backslash T^{*}\right)\right|=c$. As $f^{\prime-1}(\{t\})$ is countable for each $t \in T \backslash T^{*}$, we can select a subset $A$ of $X \cap f^{\prime-1}\left(T \backslash T^{*}\right)$ of cardinality $c$ on which $f$ acts injectively. In either case, the result follows.

LEMMA 2.3. Let $X$ be Borel-dense of order 2 in $\mathbf{R}$ and $R$ any nonreticulate member of $\mathscr{B}_{\mathbf{R} \times \mathbf{R}}$. Then $|(X \times X) \cap R|=c$.

PROOF. It suffices to show that there is a system $\left\{R_{i}: i<c\right\}$ of $c$ pairwise disjoint subsets $R_{i}$ of $R$, each being a nonreticulate member of $\mathscr{B}_{\mathbf{R} \times \mathbf{R}}$. Let $\pi_{1}\left(\pi_{2}\right)$ be the canonical projection of $R$ onto its first (second) coordinate, respectively. Let $A_{i}=\left\{x \in \mathbf{R}: \pi_{i}^{-1}(\{x\})\right\}$ is uncountable; then $A_{i}$ is analytic $(i=1,2)$. If $A_{1}$ is uncountable, we can choose a system $\left\{B_{i}: i<c\right\}$ of $c$ pairwise disjoint subsets $B_{i}$ of cardinality $c$ of $A_{1}$ with $B_{i} \in \mathscr{B}_{\mathbf{R}}$. Put $R_{i}=\pi_{1}^{-1}\left(B_{i}\right)$ for each $i<c$ to obtain the result. Therefore let us assume that $A_{1}$ and $A_{2}$ are both countable. Put $R^{*}=R \backslash\left(\left(A_{1} \times \mathbf{R}\right) \cup\left(\mathbf{R} \times A_{2}\right)\right)$. As $\pi_{1}\left(R^{*}\right)$ is uncountable and analytic, we can now choose $c$ pairwise disjoint subsets $B_{i}(i<c)$ of cardinality $c$ of $\pi_{1}\left(R^{*}\right)$ each belonging to $\mathscr{B}_{\mathbf{R}}$. Again put $R_{i}=\pi_{1}^{-1}\left(B_{i}\right)(i<c)$ to obtain the result.

If $q \in A \times B \times C \times D$ is a quadruple and, for instance, $b \in B$, we write $q=$ $(*, b, *, *)$ to denote that $q=(a, b, c, d)$ for some $a \in A, c \in C, d \in D$. Now we show:

THEOREM 2.4. Let $X$ be any Borel-dense Blackwell subset of $\mathbf{R}$. There exists a decomposition $X=\bigcup_{i<c} X_{i}$ of $X$ into c pairwise disjoint subsets $X_{i}$ with the following properties:

(1) Each subset $X_{i}(i<c)$ is Borel-dense of order 2 in $\mathbf{R}$. 
(2) Whenever $i, j<c, X_{i} \subseteq Y \subseteq X$, and $f: Y \rightarrow \mathbf{R}$ is an essential measurable function, then $\left|f\left(X_{i}\right) \cap\left(X_{j} \cup(\mathbf{R} \backslash X)\right)\right|=c$.

PROOF. Let $\left\{q_{\alpha}: \alpha<c\right\}$ be a list of all quadruples $q=(g, i, j, R)$, where $g: \mathbf{R} \rightarrow \mathbf{R}$ is an essential measurable function, the ordinals $i, j$ are less than $c$, and $R$ is a nonreticulate member of $\mathscr{B}_{\mathbf{R} \times \mathbf{R}}$. We also write $q_{\alpha}=\left(g_{\alpha}, i_{\alpha}, j_{\alpha}, R_{\alpha}\right)$. By Lemma 2.2, choose for each $\alpha<c$ a subset $A_{\alpha}$ of $X \cap \operatorname{supp}\left(g_{\alpha}\right)$ of cardinality $c$ on which $g_{\alpha}$ acts injectively. We now choose elements $x_{\alpha}, u_{\alpha}, v_{\alpha} \in \mathbf{R}(\alpha<c)$ inductively as follows. Assume that $\alpha<c$ and for each $\beta<\alpha$ we have found elements $x_{\beta} \in A_{\beta}$ and $u_{\beta}, v_{\beta} \in X$ such that $\left(u_{\beta}, v_{\beta}\right) \in R_{\beta}$ and the elements

$$
x_{\beta}, g_{\beta}\left(x_{\beta}\right), u_{\beta}, v_{\beta} \quad(\beta<\alpha)
$$

are all different from each other except that possibly $u_{\beta}=v_{\beta}$ for some $\beta<\alpha$. As $\left|A_{\alpha}\right|=c$, there exists $x_{\alpha} \in A_{\alpha}$ such that $x_{\alpha}$ and $g_{\alpha}\left(x_{\alpha}\right)$ are different from all elements listed in (*). Next, as $\left|R_{\alpha} \cap(X \times X)\right|=c$ by Lemmas 2.1 and 2.3, we can choose $u_{\alpha}, v_{\alpha} \in X$ different from $x_{\alpha}, g_{\alpha}\left(x_{\alpha}\right)$, and all elements of (*) such that $\left(u_{\alpha}, v_{\alpha}\right) \in R_{\alpha}$.

Now put

$$
\begin{aligned}
X_{i}^{\prime}= & \left\{x_{\alpha}, u_{\alpha}, v_{\alpha}: q_{\alpha}=(*, i, *, *), \alpha<c\right\} \\
& \cup\left(X \cap\left\{g_{\alpha}\left(x_{\alpha}\right): q_{\alpha}=\left(g_{\alpha}, *, i, *\right), \alpha<c\right\}\right)
\end{aligned}
$$

for each $i<c$. By construction, these sets $X_{i}^{\prime}$ are pairwise disjoint. Let $Z=X \backslash$ $\bigcup_{i<c} X_{i}^{\prime}$ and put $X_{0}=X_{0}^{\prime} \cup Z, X_{i}=X_{i}^{\prime}$ for each $0<i<c$, where $0=\min \{i: i<c\}$. Then clearly condition (1) is satisfied.

Now let $f: Y \rightarrow \mathbf{R}$ be any essential measurable mapping where $X_{i} \subseteq Y \subseteq X$, and let $i, j<c$. We can extend $f$ to a measurable function $g$ defined on all of $\mathbf{R}$ (cf. [8, p. 434]). Then $g$ is essential, and for any nonreticulate set $R$ in $\mathscr{B}_{\mathbf{R} \times \mathbf{R}}$ there exists $\alpha=\alpha(R)<c$ with $(g, i, j, R)=q_{\alpha}$. Thus $x_{\alpha} \in X_{i}$ and $g\left(x_{\alpha}\right) \in X_{j} \cup(\mathbf{R} \backslash X)$. Since there are precisely $c$ such nonreticulates $R$, we obtain $\left|f\left(X_{i}\right) \cap\left(X_{j} \cup(\mathbf{R} \backslash X)\right)\right|=c$.

Note that each superset (in $\mathbf{R}$ ) of any of the sets $X_{i}$ of Theorem 2.4 is a strong Blackwell set by Lemma 2.1. Now we give the

PROOF OF THEOREM 1. Choose a decomposition $X=\bigcup_{i \in \mathbf{R}} X_{i}$ of $X$ into $c$ pairwise disjoint subsets $X_{i}$ with all the properties of Theorem 2.4. Let $\mathscr{F}$ be the system of all unions $U_{A}=\bigcup_{i \in A} X_{i}$ where $A \subseteq \mathbf{R}$. Now suppose that $A, B$ are nonempty proper subsets of $\mathbf{R}$ and $f: U_{A} \rightarrow \mathbf{R}$ is an essential measurable function. Then $f\left(U_{A}\right)$ intersects $U_{\mathbf{R} \backslash B} \cap(\mathbf{R} \backslash X)$. Observing Lemma 2.1, it follows that $\mathscr{F}$ satisfies all the requirements of the theorem.

The following result, which is immediate both by Theorem 2.4 (and Lemma 2.1) and Theorem 1, generalizes Bhaskara Rao and Rao [1, Proposition 12] and Rosenstein [12, Theorem 9.6].

COROLlARY 2.5. Let $X$ be any Borel-dense Blackwell subset of $\mathbf{R}$ and $\kappa$ any finite or infinite cardinal number with $2 \leq \kappa \leq c$. Then there exists a decomposition $X=\bigcup_{i<\kappa} X_{i}$ of $X$ into $\kappa$ pairwise disjoint nonanalytic super-rigid strong Blackwell subsets $X_{i}$ such that, for any $i, j<\kappa$ with $i \neq j$, there is no measurable mapping of $X_{i}$ into $X_{j}$ with uncountable range.

We also note the following related result. 
COROLlaRY 2.6. Let $X$ be any Borel-dense Blackwell subset of $\mathbf{R}$. There exists a super-rigid family $\mathscr{A}$, closed under complementation in $X$, of $2^{c}$ (nonanalytic) strong Blackwell subsets of $X$ such that whenever $A, B \in \mathscr{A}$ with $A \neq B$, there exists no measurable mapping $f: A \rightarrow B$ which is either injective or surjective.

ProOF. First choose a subfield $\mathscr{F}$ of $\mathscr{P}(X)$ with the properties (1)-(3) of Theorem 1 , and let $\varphi$ be an isomorphism from $\mathscr{P}(\mathbf{R})$ onto $\mathscr{F}$. Next split $\mathbf{R}=$ $R_{1} \cup R_{2}$ into two disjoint subsets of equal cardinality, and let $f: R_{1} \rightarrow R_{2}$ be a bijection. For each subset $A \subseteq R_{1}$ put $S_{A}=A \cup\left(R_{2} \backslash f(A)\right)$. Then $\mathscr{S}=\left\{S_{A}: A \subseteq\right.$ $\left.R_{1}\right\}$ is closed under complementation, has size $2^{c}$, and consists only of pairwise incomparable elements. Now put $\mathscr{A}=\varphi(\mathscr{S})$ to obtain the result.

In view of Corollary 2.5, we wish to show that $X$ is also the union of a well ordered chain of length $c$ of pairwise nonisomorphic strong Blackwell subsets. In fact we have:

COROLlaRY 2.7. Let $X$ be any Borel-dense Blackwell subset of $\mathbf{R}$, and let $(I, \leq)$ be any linearly ordered set with $|I| \leq c$ and without a greatest or a smallest element. Then there exists a super-rigid family $\mathscr{C}$ of strong Blackwell subsets of $\mathbf{R}$ such that:

(1) $(\mathscr{C}, \subseteq)$ is a chain isomorphic to $(I, \leq)$.

(2) Whenever $A, B \in \mathscr{C}$ with $A \subsetneq B$, there is no measurable injection of $B$ into $A$, and also no measurable surjection of $A$ onto $B$.

(3) $X=\bigcup\{A: A \in \mathscr{C}\}$ and $\bigcap\{A: A \in \mathscr{C}\}=\varnothing$.

ProOF. Split $\mathbf{R}=\bigcup_{i \in I} X_{i}$ into $|I|$ pairwise disjoint nonempty subsets $X_{i}$. For each $i \in I$ let $A_{i}=\bigcup\left\{X_{j}: j \in I, j \leq i\right\}$, and put $\mathscr{A}=\left\{A_{i}: i \in I\right\}$. Clearly $(I, \leq)$ and $(\mathscr{A}, \subseteq)$ are order-isomorphic. Now let $\mathscr{C}$ be the image of $\mathscr{A}$ under any isomorphism from $\mathscr{P}(\mathbf{R})$ onto $\mathscr{F}$, where $\mathscr{F}$ is the subfield of $\mathscr{P}(X)$ of Theorem 1 . The result follows.

Let us say that two sets of reals have incomparable continuity type, if none of them is a continuous image of the other (cf. [8, p. 428]). The following observation is in the spirit of a result of Sierpiński [17] (cf. [12, Theorem 9.10(3)]) dealing with order types of subsets of $\mathbf{R}$.

COROLLARY 2.8. There are c Borel-dense subsets of $\mathbf{R}$ any two of which have incomparable continuity types but differ by only two elements.

ProOF. Let $X, Y$ be two of the subsets $X_{i}$ of $\mathbf{R}$ of Theorem 2.4. We claim that the sets $X_{y}=X \cup\{y\}(y \in Y)$ satisfy our requirements. Suppose that $y, z \in Y$ with $y \neq z$ and $f: X_{y} \rightarrow X_{z}$ is continuous and onto. Let $x \in X_{y}$ with $f(x)=z$. There is a neighborhood $U$ of $x$ in $X_{y}$ which is disjoint with $f(U)$. Hence $U \backslash\{y\} \subseteq$ $f(\operatorname{supp}(f))$. As $X$ is Borel-dense, $U$ is uncountable and $f$ is essential. But then $\left|X_{z} \cap Y\right|=c$ by Theorem 2.4, a contradiction.

\section{REFERENCES}

1. K. P. S. Bhaskara Rao and B. V. Rao, Borel spaces, Dissertationes Math. (Rozprawy Mat.) 190 (1981), 1-63.

2. K. P. S. Bhaskara Rao and R. M. Shortt, Generalised Lusin sets with the Blackwell property, Fund. Math. 127 (1986), 9-39. 
3. D. Blackwell, On the class of probability spaces, Proc. Third Berkeley Sympos. on Mathematical Statistics and Probability, vol. 2, Univ. of California Press, 1956, pp. 1-6.

4. M. Dugas and R. Göbel, Every cotorsion-free algebra is an endomorphism algebra, Math. Z. 181 (1982), 451-470.

5. D. Fremlin, On Blackwell algebras (unpublished manuscript, 1980).

6. J. Hoffman-Jørgensen, The theory of analytic spaces, Various publication series, no. 10, Aarhus Univ., 1970.

7. J. Jasiński, On the combinatorial properties of Blackwell sets, Proc. Amer. Math. Soc. 93 (1985), $657-660$.

8. K. Kuratowski, Topology, vol. I, Academic Press, New York; PWN, Warszawa, 1966.

9. G. W. Mackey, Borel structures in groups and their duals, Trans. Amer. Math. Soc. 85 (1957), 134-165.

10. M. Orkin, A Blackwell space which is not analytic, Bull. Acad. Polon. Sci. 20 (1972), 437-438.

11. D. Ramachandran, Perfect measures, Macmillan-India, New Delhi, 1979.

12. J. Rosenstein, Linear orderings, Academic Press, New York, 1982.

13. H. Sarbadhikari, Some contributions to descriptive set theory, Thesis, Indian Statistical Institute, Calcutta, 1977.

14. R. M. Shortt, Borel-dense Blackwell spaces are strongly Blackwell, Colloq. Math. 53 (1987), 35-41.

15. __ Notions of independence for random variables, Probab. Math. Stat. (to appear).

16. $\ldots$, A separation principle for Blackwell sets, Bull. Polon. Acad. Sci. Math. 34 (1986), 643645.

17. W. Sierpiński, Sur les types d'ordre des ensembles linéaires, Fund. Math. 37 (1950), 253-264.

FAChBEREICh 6-MAThematik, UNiVersität GHS ESSEN, 4300 ESSEN 1, West GeRMANY 